\title{
Neutrophil-lymphocyte ratio correlates with limited joint mobility of hand in patients with type 2 diabetes
}

\author{
Yusuke Mineoka ${ }^{1)}$, Michiyo Ishii ${ }^{1)}$, Yoshitaka Hashimoto ${ }^{2)}$, Naoto Nakamura ${ }^{3)}$, Yasukazu Katsumi ${ }^{4)}$, \\ Motohide Isono $^{1)}$ and Michiaki Fukui ${ }^{2}$ \\ 1) Department of Internal Medicine, Otsu Municipal Hospital, Otsu, Japan \\ 2) Department of Endocrinology and Metabolism, Kyoto Prefectural University of Medicine, Graduate School of Medical Science, \\ Kyoto, Japan \\ 3) Department of Internal Medicine, Saiseikai Kyoto Hospital, Nagaokakyo, Japan \\ 4) Department of Orthopedics, Uji Takeda Hospital, Uji, Japan
}

\begin{abstract}
Limited joint mobility (LJM) of hand, which is one of the complications of diabetic hand, is associated with diabetic micro- and macroangiopathy although the precise pathogenesis is not completely understood. Neutrophil-lymphocyte ratio (NLR), a simple and novel inflammatory marker, has been reported to have a predictive effect to some diabetic complications in recent years. However, it is not elucidated about the relationship between LJM of hand and NLR in patients with type 2 diabetes. We evaluated the relationships between LJM of hand and NLR in 335 consecutive patients with type 2 diabetes in this cross-sectional study. LJM of hand was diagnosed by a 'prayer sign' or 'table test'. LJM of hand was present in 80 patients. The patients with LJM of hand had significantly older age, longer duration of diabetes, worse renal function, and higher proportion of diabetic neuropathy, retinopathy and nephropathy. NLR in patients with LJM of hand was higher than that in patients without LJM of hand $(2.54 \pm 1.46$ vs. $2.11 \pm 1.04, p=0.004)$. Multivariate logistic regression analysis revealed that LJM of hand was positively correlated with NLR (odds ratio, 1.31; 95\% confidence interval 1.03-1.69, $p=$ 0.027) after adjustment for age, sex, duration of diabetes, body mass index, hemoglobin A1c, hypertension and dyslipidemia. Our results demonstrate a positive relation between LJM of hand and NLR in patients with type 2 diabetes.
\end{abstract}

Key words: Clinical practice, Neutrophil-lymphocyte ratio, Diabetic hand, Limited joint mobility

'DIABETIC HAND', which manifests as conditions such as limited joint mobility (LJM), Dupuytren's contracture and trigger finger, is a complication of the upper extremity in patients with diabetes [1-5]. Diabetic hand tends to be ignored in clinical situations [6] in spite of several studies reporting the association between LJM of hand and micro- $[1-4,7]$ and macroangiopathy $[8,9]$. The precise etiology of LJM of hand is not completely understood although multi-factorial reasons were hypothesized such as a genetic component, increased nonenzymatic collagen glycosylation and polyol pathway due to chronic hyperglycemia $[4,5,10]$.

Type 2 diabetes mellitus is thought to be an inflamma-

Submitted Apr. 4, 2018; Accepted Jun. 21, 2018 as EJ18-0143 Released online in J-STAGE as advance publication Jul. 14, 2018 Correspondence to: Michiaki Fukui M.D. Ph.D., Department of Endocrinology and Metabolism, Kyoto Prefectural University of Medicine, Graduate School of Medical Science, 465 Kajii-cho, Kawaramachi-Hirokoji, Kamigyo-ku, Kyoto 602-8566, Japan. E-mail: sayarinapm@hotmail.com tory disease triggered by disordered metabolism [11] Hyperglycemia and micro-inflammation are considered to be related to diabetes and its complications [12]. A raised white blood cell (WBC) is reported to be associated with higher risk of type 2 diabetes [13]. Neutrophils indicate the active nonspecific inflammatory mediator, which leads to ischemic injury [14], whereas lymphocytes indicate the protective component of inflammation [15].

Neutrophil-lymphocyte ratio (NLR), which can be calculated easily using peripheral blood count, has a close association with inflammation, which related to cancer and cardiovascular disease [16-18]. In addition, it has also been reported that NLR is associated with insulin resistance [19] and diabetic retinopathy and nephropathy $[20,21]$. However, to our best of knowledge, there are no reports about the relationship between NLR and diabetic hand in patients with type 2 diabetes. Therefore, we investigated the relationship between NLR and LJM of hand in this cross-sectional study. 


\section{Research Design and Methods}

\section{Study design and patients}

This study was conducted in accordance with the Declaration of Helsinki and informed consent was obtained from all patients. Ethics Committee of Otsu Municipal Hospital gave approval for this study. In this study, we included the patients who were examined the LJM of hand, from April 2012 to December 2014 at outpatient clinic of Otsu Municipal Hospital. Patients with a history of articular rheumatism or surgery for spinal disease were excluded. Patients with acute inflammatory or infectious disease, severe tissue damage, acute massive hemorrhage, malignancies or hematologic diseases which affect neutrophils and lymphocytes, were also excluded.

All patients provided details of their demographics and medical history. According to self administered questionnaire, patients were classified as current smokers or not. Body mass index (BMI) was calculated as weight in kilograms divided by height in meters squared. Type 2 diabetes was diagnosed according to the Report of the Expert Committee on the Diagnosis and Classification of Diabetes Mellitus [22].

Neuropathy was defined using the diagnostic criteria for diabetic neuropathy proposed by the Diagnostic Neuropathy Study Group [23]. Diabetic neuropathy was diagnosed as follows: neuropathic symptoms such as decreased or absent ankle reflex (bilateral); neuropathic pain, paresthesia and numbness and decreased distal sensation assessed by $\mathrm{C} 128 \mathrm{~Hz}$ tuning fork without evident non-diabetic peripheral neuropathy.

Retinopathy was assessed by ophthalmologists blinded to the severity of other diabetic complications and was graded as proliferative diabetic retinopathy (PDR) or non-PDR according to the International Clinical Diabetic Retinopathy Disease Severity Scale [24]. If findings in the left and right fundi were discordant, the worse side was taken as representative of the patient. Nephropathy was graded as normoalbuminuria, urine albumin excretion (UAE) $<30 \mathrm{mg} / \mathrm{g}$ creatinine $(\mathrm{Cr}$ ); microalbuminuria, 30-300 mg/g Cr; or macroalbuminuria, $>300 \mathrm{mg} / \mathrm{g} \mathrm{Cr}$ [25]. Microalbuminuria and macroalbuminuria were considered to indicate nephropathy.

LJM of hand was objectively recorded and diagnosed using a 'prayer sign' or 'table test', which is commonly used for assessment of LJM. We asked the patients to close their palms in a praying position with the fingers in contact with each other or to place the palms on a table with the wrists flexed maximally. Patients with LJM of hand are unable to bring the palms together or place them on a table with full contact [2-4].

\section{Data collection and measurements}

Blood samples were drawn in the morning after an overnight fast for measurement of blood count (including neutrophil and lymphocytes), glycated hemoglobin (HbA1c), total cholesterol (TC), triglycerides (TG), uric acid (UA) and creatinine. Complete blood count and examinations were performed using a Beckman Coulter LH 780 instrument and Bio Majesty JCA-BM 6050 (JEOL, Tokyo, Japan).

NLRs were calculated as the ratio of the absolute neutrophil and lymphocyte counts. Total white blood cells (WBCs), neutrophils and lymphocytes, were obtained from the same automated blood sample. Hypertension was defined as systolic blood pressure (SBP) $>140$ $\mathrm{mmHg}$, diastolic blood pressure (DBP) $>90 \mathrm{mmHg}$, and/or having received treatment for hypertension. Dyslipidemia was defined as a TC concentration $>5.69$ $\mathrm{mmol} / \mathrm{L}$, a TG concentration $>1.70 \mathrm{mmol} / \mathrm{L}$, and/or having received treatment for dyslipidemia.

The estimated glomerular filtration rate (eGFR) was used to estimate kidney function and was calculated as follows [26]: Male: eGFR $\left(\mathrm{mL} \cdot \mathrm{min}^{-1} \cdot 1.73 \mathrm{~m}^{-2}\right)=0.741$ $\times 175 \times$ age $^{-0.203} \times(\text { serum creatinine })^{-1.154}$. Female $:$ $\mathrm{eGFR}=0.741 \times 175 \times$ age $^{-0.203} \times(\text { serum creatinine })^{-1.154}$ $\times 0.742$.

\section{Statistical analysis}

Statistical analyses were performed using JMP v. 9.0 (SAS Institute Inc., Cary, NC). A $p$ value $<0.05$ was considered significant. Continuous variables were presented as the mean value $\pm 1 \mathrm{SD}$. Categorical variables were presented as a number (percentage).

The significance of differences between groups was evaluated by unpaired Student $t$-test or Pearson $\chi^{2}$ test. Pearson's correlation analyses were used to assess the relationships between NLR and the other variables. Univariate and multivariate logistic regression analyses were used to investigate the relationship between LJM of hand and NLR. In multivariate logistic regression analysis, factors which were found the association in univariate analysis and which had been known the association with diabetic hand, were selected for covariates; age, sex, duration of diabetes, BMI, HbA1c, hypertension, and dyslipidemia. 


\section{Results}

LJM was examined in 416 consecutive patients with type 2 diabetes. Of these patients, 81 were excluded because of histories of rheumatoid arthritis or surgery for spinal disease $(N=40)$, acute inflammatory or infectious disease $(N=3)$, severe tissue damage $(N=12)$, acute massive hemorrhage $(N=2)$, and malignancies or hematologic diseases $(N=24)$. A total of 335 patients with type 2 diabetes were included in this study. The clinical characteristics of patients (187 male and 148 female) with type 2 diabetes are shown in Table 1. LJM of hand was present in 80 patients, and these patients had significantly older age, longer duration of diabetes, worse renal function, and higher proportion of diabetic neuropathy, retinopathy and nephropathy. Lymphocyte was significantly lower in patients with LJM of hand $(2,093 \pm 931$ vs. $1,865 \pm 765, p=0.049$ ) whereas neutrophil was not different between groups $(3,665 \pm 1,605$ vs. 3,624 $\pm 1,853, p=0.848$ ), then NLR in patients with LJM of hand was higher than that in patients without LJM of hand $(2.54 \pm 1.46$ vs. $2.11 \pm 1.04, p=0.004)$ (Table 1). We performed the comparison analyses between NLR and several variables including the presence of neuropathy, retinopathy and neuropathy. NLR was significantly higher in the groups of hypertension, neuropathy, retinopathy and neuropathy (Table 2). In univariate logistic regression analyses, LJM of hand was correlated with age, male sex, duration of diabetes, BMI, hypertension, dyslipidemia, microangiopathy and NLR. Multivariate logistic regression analysis revealed that LJM of hand was positively correlated with NLR (OR, 1.31; 95\% CI, 1.03-1.69) after adjustment for age, sex, duration of diabetes, BMI, HbAlc, hypertension, and dyslipidemia (Table 3).

Table 1 Clinical characteristics of patients

\begin{tabular}{|c|c|c|c|}
\hline & $\begin{array}{l}\mathrm{LJM}(-) \\
(n=255)\end{array}$ & $\begin{array}{c}\mathrm{LJM}(+) \\
(n=80)\end{array}$ & $p$ \\
\hline Age (years) & $65.7 \pm 12.7$ & $72.8 \pm 11.1$ & $<0.001$ \\
\hline Male $(\%)$ & 39.6 & 58.7 & 0.003 \\
\hline $\operatorname{BMI}\left(\mathrm{kg} / \mathrm{m}^{2}\right)$ & $25.0 \pm 4.9$ & $23.7 \pm 4.9$ & 0.046 \\
\hline Hemoglobin A1c (\%) & $7.4 \pm 1.1$ & $7.7 \pm 1.2$ & 0.074 \\
\hline Duration of type 2 diabetes (year) & $14.8 \pm 8.9$ & $20.6 \pm 10.5$ & $<0.001$ \\
\hline $\mathrm{eGFR}\left(\mathrm{mL} / \mathrm{min} / 1.73 \mathrm{~m}^{2}\right)$ & $68.3 \pm 23.4$ & $59.7 \pm 20.4$ & 0.005 \\
\hline Creatinine $(\mu \mathrm{mol} / \mathrm{L})$ & $77.4 \pm 31.5$ & $72.3 \pm 25.6$ & 0.154 \\
\hline Systolic blood pressure $(\mathrm{mmHg})$ & $132.5 \pm 18.2$ & $132.8 \pm 19.4$ & 0.850 \\
\hline Dyastlic blood pressure (mmHg) & $74.8 \pm 52.9$ & $67.6 \pm 12.9$ & 0.241 \\
\hline Total cholesterol (mmol/L) & $4.55 \pm 0.83$ & $4.56 \pm 0.88$ & 0.980 \\
\hline Triglyceride $(\mathrm{mmol} / \mathrm{L})$ & $1.71 \pm 1.07$ & $1.78 \pm 1.35$ & 0.605 \\
\hline WBC $\left(/ \mathrm{mm}^{3}\right)$ & $6.69 \pm 1.88$ & $6.8 \pm 2.16$ & 0.656 \\
\hline Neutrophil $\left(/ \mathrm{mm}^{3}\right)$ & $3,665 \pm 1,605$ & $3,624 \pm 1,853$ & 0.848 \\
\hline Lymphocyte $\left(/ \mathrm{mm}^{3}\right)$ & $2,093 \pm 931$ & $1,865 \pm 765$ & 0.049 \\
\hline NLR & $2.11 \pm 1.04$ & $2.54 \pm 1.46$ & 0.004 \\
\hline Hypertension (\%) & 62.7 & 82.5 & 0.001 \\
\hline Dyslipidemia (\%) & 59.2 & 73.7 & 0.019 \\
\hline Neuropathy (\%) & 31.3 & 65.0 & $<0.001$ \\
\hline Retinopathy (\%) & 25.5 & 51.3 & $<0.001$ \\
\hline Nephropathy (\%) & 42.7 & 67.5 & $<0.001$ \\
\hline
\end{tabular}

Continuous variables are presented as the mean value $\pm 1 \mathrm{SD}$. Categorical variables are presented as a number (percentage). LJM, limited joint mobility; BMI, body mass index; eGFR, estimated glomerular filtration rate; WBC, white blood cell; NLR, neutrophil-lymphocyte ratio. 
Table 2 NLR in several clinical variables

\begin{tabular}{lcc}
\hline & NLR & $p$ \\
\hline Sex (male/female) & $2.01 \pm 0.78 / 1.99 \pm 0.75$ & 0.863 \\
\hline Hypertension $(-/+)$ & $1.85 \pm 0.71 / 2.07 \pm 0.84$ & 0.015 \\
Dyslipidemia $(-/+)$ & $1.97 \pm 0.71 / 2.02 \pm 0.80$ & 0.597 \\
\hline Neuropathy $(-/+)$ & $1.92 \pm 0.76 / 2.13 \pm 0.77$ & 0.019 \\
Retinopathy $(-/+)$ & $1.94 \pm 0.68 / 2.14 \pm 0.91$ & 0.037 \\
\hline Nephropathy $(-/+)$ & $1.87 \pm 0.69 / 2.15 \pm 0.82$ & 0.001 \\
\hline
\end{tabular}

Continuous variables are presented as the mean value $\pm 1 \mathrm{SD}$. NLR, neutrophil-lymphocyte ratio.

\section{Discussion}

In this study, we evaluated the relationships between LJM of hand and NLR in patients with type 2 diabetes and multivariate logistic regression analysis indicated that LJM of hand was correlated with NLR after adjusted for several factors. To the best of our knowledge, this is the first report investigate the relationship between LJM of hand and NLR. LJM of hand is one of the complications of 'Diabetic hand', which is tended to be overlooked [6]. Relationship between diabetic hand especially LJM of hand, and micro and macro vascular complications were previously reported $[1-6,8,9]$. NLR, including components both neutrophils and lymphocytes, has been recently described as a simple and novel inflammatory marker in cancer and cardiovascular disea- ses $[16,18,27,28]$. Elevated NLR levels were correlated with an increased risk of developing hypertension [29]. NLR was related to the prevalence and incidence of type 2 diabetes and insulin resistance [19]. Furthermore, NLR is reported to be associated with microvascular complications $[20,21,30,31]$. Previous report indicated that increased NLR was significantly associated with diabetic nephropathy, and high NLR values might be a reliable predictive marker of early-stage diabetic nephropathy [21]. Other study reported that patients with diabetic retinopathy had increased NLR values compared with diabetic patients who did not have retinopathy [30]. NLR was related to the prevalence and severity of coronary arterial disease [32]. In this study, multivariate logistic regression analysis indicated that LJM of hand was correlated with NLR after adjusted for several factors. In recent year, positive association between NLR and diabetic peripheral neuropathy (DPN) is reported [33, 34]. The relation between LJM of hand and NLR might be similar to some extent with the microvascular complications including DPN which were well-known comorbidities of LJM of hand. The precise pathogenesis of LJM of hand is incompletely understood although several studies have revealed the relationship between chronic inflammation and diabetes and its complications $[12,35,36]$. NLR including two different immune system; neutrophils which are related to ongoing inflammation [14] and lymphocytes which reflected the immune regulatory pathway [15]. NLR represents the balance between neu-

Table 3 Univariate and multivariate-adjusted ORs (95\% CI) for risk factors associated with LJM of hand

\begin{tabular}{|c|c|c|c|c|}
\hline & \multicolumn{2}{|c|}{ Univariate analysis } & \multicolumn{2}{|c|}{ Multivariate analysis } \\
\hline & OR $(95 \% \mathrm{CI})$ & $p$ & OR $(95 \% \mathrm{CI})$ & $p$ \\
\hline Age & $1.05(1.03-1.08)$ & $<0.001$ & $1.04(1.01-1.07)$ & 0.009 \\
\hline Male & $2.39(1.42-4.06)$ & 0.001 & $2.04(1.10-3.79)$ & 0.023 \\
\hline Duration of diabetes & $1.06(1.03-1.09)$ & $<0.001$ & $1.04(1.01-1.07)$ & 0.023 \\
\hline BMI & $0.94(0.89-0.99)$ & 0.042 & $0.98(0.91-1.05)$ & 0.633 \\
\hline Hemoglobin A1c & $1.21(0.98-1.49)$ & 0.080 & $1.30(1.00-1.70)$ & 0.051 \\
\hline Creatinine & $0.99(0.59-1.43)$ & 0.988 & - & - \\
\hline Smoking & $0.69(0.32-1.59)$ & 0.374 & - & - \\
\hline Hypertension & $2.79(1.53-5.44)$ & 0.001 & $1.63(0.74-3.81)$ & 0.226 \\
\hline Dyslipidemia & $1.94(1.12-3.45)$ & 0.017 & $1.43(0.71-2.99)$ & 0.319 \\
\hline NLR & $1.33(1.09-1.64)$ & 0.006 & $1.31(1.03-1.69)$ & 0.027 \\
\hline
\end{tabular}

Data are expressed as mean \pm SD. LJM, limited joint mobility; BMI, body mass index; eGFR, estimated glomerular filtration rate; NLR, neutrophil-lymphocyte ratio. 
trophils and lymphocyte, and is thought to be an indicator of systemic inflammation [17]. This might partially explain the reason why NLR correlated LJM of hand. Inflammation has an important role in the development of vascular complications in diabetic patients, therefore diabetes and its complications had been demonstrated as an inflammatory disease $[11,12]$. Formation of advanced glycosylation end-products (AGEs), hyperglycemia and oxidative stress were also thought to be related with the development of LJM of hand [37-39]. In this study, we identified that NLR correlated with LJM of hand in patients with type 2 diabetes. LJM of hand, which is associated with diabetic micro- $[1-4,7]$ and macrocomplications $[8,9,40]$, is relatively easy to notify; however, clinicians often overlook this complication of diabetes in clinical situations [6]. NLR, which calculated simply and easily in routine clinical practice, could be used as a marker of diabetic complications including LJM of hand.

This study has several limitations. First, the crosssectional design only permits inference of the causal relationships between NLR and LJM of hand. Second, we did not investigate other diabetic hand such as Dupuytren's contracture or carpal tunnel syndrome. However, it is likely that these hand syndromes are also associated with NLR because the same pathologies such as microvascular disease or abnormal collagen deposition are hypothesized [38]. Third, we did not evaluate NLR with other inflammatory mediators such as high sensitivity C-reactive protein (hs-CRP), tumor necrosis factor-alpha and interleukin 6 , so we could not detect whether NLR was a better inflammatory marker in this research although previous report indicated that NLR correlated with hs-CRP and had a specific role to detect DPN rather than other inflammation factors [33]. Finally, the patients in this study were all Japanese, and it is uncertain if these findings are general.

In summary, we investigated the positive relationship between NLR and LJM of hand in patients with type 2 diabetes. Further study is needed to evaluate the application for NLR to predict LJM of hand in patients with type 2 diabetes.

\section{Acknowledgments}

We thank all staff members of the diabetes team in Otsu Municipal Hospital for their assistance.

\section{Conflict of Interest}

Michiaki Fukui has received grants, honoraria and research supports from AstraZeneca plc., Astellas Pharma Inc., Nippon Boehringer Ingelheim Co., Ltd., Daiichi Sankyo Co., Ltd., Eli Lilly Japan K.K., Kyowa Hakko Kirin Company Ltd., Kissei Pharmaceutical Co., Ltd., MSD K.K., Mitsubishi Tanabe Pharma Corporation, Novo Nordisk Pharma Ltd., Sanwa Kagaku Kenkyusho Co., Ltd., Sanofi K.K., Ono Pharmaceutical Co., Ltd., and Takeda Pharmaceutical Co., Ltd. The sponsors were not involved in the study design; in the collection, analysis, interpretation of data; in the writing of this manuscript; or in the decision to submit the article for publication. The authors, their immediate families, and any research foundations with which they are affiliated have not received any financial payments or other benefits from any commercial entity related to the subject of this article. The authors declare that although they are affiliated with a department that is supported financially by pharmaceutical company, the authors received no current funding for this study and this does not alter their adherence to all the journal policies on sharing data and materials. The other authors have nothing to disclose.

\section{Contributors}

YM contributed to the conception and design, wrote the manuscript, and researched and analyzed data. MI contributed to acquisition of data, critical revision of the manuscript, and analysis and interpretation of data, and approved the final version of the manuscript. YH contributed to critical revision of the manuscript, and to analysis and interpretation of data. NN and YK contributed to revision of the manuscript. MI contributed to acquisition of data and reviewed the manuscript. MF contributed to critical revision of the manuscript.

\section{References}

1. Rosenbloom AL, Silverstein JH, Lezotte DC, Richardson K, McCallum M (1981) Limited joint mobility in childhood diabetes mellitus indicates increased risk for micro- vascular disease. N Engl J Med 305: 191-194.

2. Rosenbloom AL (1989) Limitation of finger joint mobility in diabetes mellitus. $J$ Diabet Complications 3: 77-87. 
3. Arkkila PE, Kantola IM, Viikari JS, Rönnemaa T, Vähätalo MA (1996) Limited joint mobility is associated with the presence but does not predict the development of microvascular complications in type 1 diabetes. Diabet Med 13: 828-833.

4. Crispin JC, Alcocer-Varela J (2003) Rheumatologic manifestations of diabetes mellitus. Am J Med 114: 753-757.

5. Jennings AM, Milner PC, Ward JD (1989) Hand abnormalities are associated with the complications of diabetes in type 2 diabetes. Diabet Med 6: 43-47.

6. Papanas N, Maltezos E (2010) The diabetic hand: a forgotten complication? J Diabetes Complications 24: 154162.

7. Mineoka Y, Ishii M, Tsuji A, Komatsu Y, Katayama Y, et al. (2017) Relationship between limited joint mobility of the hand and diabetic foot risk in patients with type 2 diabetes. J Diabetes 9: 628-633.

8. Arkkila PE, Kantola IM, Viikari JS (1997) Limited joint mobility in non-insulin-dependent diabetic (NIDDM) patients: correlation to control of diabetes, atherosclerotic vascular disease, and other diabetic complications. J Diabetes Complications 11: 208-217.

9. Frost D, Beischer W (2001) Limited joint mobility in type 1 diabetic patients: associations with microangiopathy and subclinical macroangiopathy are different in men and women. Diabetes Care 24: 95-99.

10. Smith LL, Burnet SP, McNeil JD (2003) Musculoskeletal manifestations of diabetes mellitus. Br J Sports Med 37: 30-35.

11. Donath MY, Shoelson SE (2011) Type 2 diabetes as an inflammatory disease. Nat Rev Immunol 11: 98-107.

12. Wada J, Makino H (2013) Inflammation and the pathogenesis of diabetic nephropathy. Clin Sci (Lond) 124: 139152.

13. Gkrania-Klotsas E, Ye Z, Cooper AJ, Sharp SJ, Luben R, et al. (2010) Differential white blood cell count and type 2 diabetes: systematic review and meta-analysis of crosssectional and prospective studies. PLoS One 5: e13405.

14. Buck BH, Liebeskind DS, Saver JL, Bang OY, Yun SW, et al. (2008) Early neutrophilia is associated with volume of ischemic tissue in acute stroke. Stroke 39: 355-360.

15. Bhutta H, Agha R, Wong J, Tang TY, Wilson YG, et al. (2011) Neutrophil-lymphocyte ratio predicts medium-term survival following elective major vascular surgery: a cross-sectional study. Vasc Endovascular Surg 45: 227231.

16. Rudiger A, Burckhardt OA, Harpes P, Müller SA, Follath F (2006) The relative lymphocyte count on hospital admission is a risk factor for long-term mortality in patients with acute heart failure. Am J Emerg Med 24: 451-454.

17. Tamhane UU, Aneja S, Montgomery D, Rogers EK, Eagle KA, et al. (2008) Association between admission neutro- phil to lymphocyte ratio and outcomes in patients with acute coronary syndrome. Am J Cardiol 102: 653-657.

18. Yamanaka $\mathrm{T}$, Matsumoto $\mathrm{S}$, Teramukai S, Ishiwata $\mathrm{R}$, Nagai Y, et al. (2007) The baseline ratio of neutrophils to lymphocytes is associated with patient prognosis in advanced gastric cancer. Oncology 73: 215-220.

19. Lou M, Luo P, Tang R, Peng Y, Yu S, et al. (2015) Relationship between neutrophil-lymphocyte ratio and insulin resistance in newly diagnosed type 2 diabetes mellitus patients. BMC Endocr Disord 15: 9.

20. Yue S, Zhang J, Wu J, Teng W, Liu L, et al. (2015) Use of the monocyte-to-lymphocyte ratio to predict diabetic retinopathy. Int J Environ Res Public Health 12: 1000910019.

21. Huang W, Huang J, Liu Q, Lin F, He Z, et al. (2015) Neutrophil-lymphocyte ratio is a reliable predictive marker for early-stage diabetic nephropathy. Clin Endocrinol (Oxf) 82: 229-233.

22. American Diabetes Association (2012) Diagnosis and classification of diabetes mellitus. Diabetes Care 35 Suppl 1: S64-S71.

23. Yasuda H, Sanada M, Kitada K, Terashima T, Kim H, et al. (2007) Rationale and usefulness of newly devised abbreviated diagnostic criteria and staging for diabetic polyneuropathy. Diabetes Res Clin Pract 77 Suppl 1: S178-S183.

24. Wilkinson CP, Ferris FL 3rd, Klein RE, Lee PP, Agardh CD, et al. (2003) Proposed international clinical diabetic retinopathy and diabetic macular edema disease severity scales. Ophthalmology 110: 1677-1682.

25. Hashimoto $\mathrm{Y}$, Tanaka M, Senmaru T, Okada H, Hamaguchi M, et al. (2015) Heart rate-corrected QT interval is a novel risk marker for the progression of albuminuria in people with type 2 diabetes. Diabet Med 32: 12211226.

26. Matsuo S, Imai E, Horio M, Yasuda Y, Tomita K, et al. (2009) Revised equations for estimated GFR from serum creatinine in Japan. Am J Kidney Dis 53: 982-992.

27. McMillan DC (2009) Systemic inflammation, nutritional status and survival in patients with cancer. Curr Opin Clin Nutr Metab Care 12: 223-226.

28. Núñez J, Núñez E, Bodí V, Sanchis J, Miñana G, et al. (2008) Usefulness of the neutrophil to lymphocyte ratio in predicting long-term mortality in ST segment elevation myocardial infarction. Am J Cardiol 101: 747-752.

29. Liu X, Zhang Q, Wu H, Du H, Liu L, et al. (2015) Blood neutrophil to lymphocyte ratio as a predictor of hypertension. Am J Hypertens 28: 1339-1346.

30. Ulu SM, Dogan M, Ahsen A, Altug A, Demir K, et al. (2013) Neutrophil-to-lymphocyte ratio as a quick and reliable predictive marker to diagnose the severity of diabetic retinopathy. Diabetes Technol Ther 15: 942-947.

31. Öztürk ZA, Kuyumcu ME, Yesil Y, Savas E, Y1ldız H, et 
al. (2013) Is there a link between neutrophil-lymphocyte ratio and microvascular complications in geriatric diabetic patients? J Endocrinol Invest 36: 593-599.

32. Verdoia M, Schaffer A, Barbieri L, Aimaretti G, Marino P, et al. (2015) Impact of diabetes on neutrophil-tolymphocyte ratio and its relationship to coronary artery disease. Diabetes Metab 41: 304-311.

33. Liu S, Zheng H, Zhu X, Mao F, Zhang S, et al. (2017) Neutrophil-to-lymphocyte ratio is associated with diabetic peripheral neuropathy in type 2 diabetes patients. Diabetes Res Clin Pract 130: 90-97.

34. Xu T, Weng Z, Pei C, Yu S, Chen Y, et al. (2017) The relationship between neutrophil-to-lymphocyte ratio and diabetic peripheral neuropathy in Type 2 diabetes mellitus. Medicine (Baltimore) 96: e8289.

35. Pitsavos C, Tampourlou M, Panagiotakos DB, Skoumas Y, Chrysohoou C, et al. (2007) Association between lowgrade systemic inflammation and type 2 diabetes mellitus among men and women from the ATTICA study. Rev Dia- bet Stud 4: 98-104.

36. Fujita T, Hemmi S, Kajiwara M, Yabuki M, Fuke Y, et al. (2013) Complement-mediated chronic inflammation is associated with diabetic microvascular complication. Diabetes Metab Res Rev 29: 220-226.

37. Goldin A, Beckman JA, Schmidt AM, Creager MA (2006) Advanced glycation end products: sparking the development of diabetic vascular injury. Circulation 114: 597605.

38. Arkkila PE, Gautier JF (2003) Musculoskeletal disorders in diabetes mellitus: an update. Best Pract Res Clin Rheumatol 17: 945-970.

39. Gerrits EG, Landman GW, Nijenhuis-Rosien L, Bilo HJ (2015) Limited joint mobility syndrome in diabetes mellitus: a minireview. World J Diabetes 6: 1108-1112.

40. Mineoka Y, Ishii M, Hashimoto Y, Tanaka M, Nakamura $\mathrm{N}$, et al. (2017) Relationship between limited joint mobility of hand and carotid atherosclerosis in patients with type 2 diabetes. Diabetes Res Clin Pract 132: 79-84. 